\title{
Semantic Interaction for Sensemaking: Inferring Analytical Reasoning for Model Steering
}

\author{
Alex Endert, Patrick Fiaux, and Chris North, Member, IEEE
}

\begin{abstract}
Visual analytic tools aim to support the cognitively demanding task of sensemaking. Their success often depends on the ability to leverage capabilities of mathematical models, visualization, and human intuition through flexible, usable, and expressive interactions. Spatially clustering data is one effective metaphor for users to explore similarity and relationships between information, adjusting the weighting of dimensions or characteristics of the dataset to observe the change in the spatial layout. Semantic interaction is an approach to user interaction in such spatializations that couples these parametric modifications of the clustering model with users' analytic operations on the data (e.g., direct document movement in the spatialization, highlighting text, search, etc.). In this paper, we present results of a user study exploring the ability of semantic interaction in a visual analytic prototype, ForceSPIRE, to support sensemaking. We found that semantic interaction captures the analytical reasoning of the user through keyword weighting, and aids the user in co-creating a spatialization based on the user's reasoning and intuition.
\end{abstract}

Index Terms - User Interaction, visualization, sensemaking, analytic reasoning, visual analytics.

\section{INTRODUCTION}

Visual analytics is a science based on supporting sensemaking of large, complex datasets through interactive visual data exploration [2]. The success of such systems hinges on their ability to combine capabilities of statistical models, visualization, and human intuition with the goal of supporting the user's analytic process. Through interacting with the system, users are able to explore possible connections, investigate hypotheses, and ultimately gain insight. This complex and personal process is referred to as sensemaking [3].

Sensemaking is composed of two primary parts - foraging and synthesis. Foraging refers to the stages of the process where users filter and gather collections of interesting or relevant information, while synthesis describes stages of the process where users create and test hypotheses about how foraged information may relate. In general, foraging lends itself to more computational support, while synthesis leverages human intuition for establishing relationships between information. Thus, a goal of visual analytics is to develop visualizations that are tightly coupled with mathematical models to provide computational support for the user - integrating foraging and synthesis.

Semantic interaction $[1,4]$ is an approach that enables such coupling, where analytic interactions designed for synthesis in visualizations are also designed to steer the underlying computation responsible for foraging of relevant information. Semantic interaction focuses on enabling direct manipulation of spatializations, which are two-dimensional views of highdimensional data such that similarity between information is represented by relative distances between data points (e.g., a cluster represents a collection of similar information) [5].

ForceSPIRE (shown in Fig. 1) is a visual analytic prototype incorporating semantic interaction for analysis of text document collections represented in a spatialization [1]. Semantic interactions

\footnotetext{
- Alex Endert is with Virginia Tech, e-mail: aendert@vt.edu.

- Patrick Fiaux is with Virginia Tech, e-mail:pfiaux@vt.edu.

- Chris North is with Virginia Tech, e-mail: north@vt.edu
}

Manuscript received 31 March 2012; accepted 1 August 2012; posted online 14 October 2012; mailed on 5 October 2012.

For information on obtaining reprints of this article, please send e-mail to:tvcg@computer.org. in ForceSPIRE include repositioning documents, highlighting text, searching, and annotating documents. When users perform semantic interactions in the course of their reasoning process, the system incrementally updates a keyword weighting scheme in accordance with the user's analytical reasoning (Table 1). The learned weighting scheme emphasizes relevant keyword entities within the dataset and adjusts the layout of the spatialization accordingly. Thus, the goal of ForceSPIRE is to automatically steer the spatialization based on the user's interaction with a subset of the information. Essentially, human and computer co-create the spatial layout.

The challenge, then, is deriving a weighting scheme representative of the user's analytical reasoning, and ensuring that the co-creation of the spatialization is in accordance with the user's reasoning process and intended meaning of the layout. Further, the semantic interactions should enable users to cognitively focus on their analysis rather than on directly steering a complex mathematical spatialization model.

In this paper we present the results of a user study exploring ForceSPIRE's ability to address these challenges. How can ForceSPIRE systematically quantify the reasoning process of users by building and modifying an entity weighting scheme? Additionally, we explore if the weighting scheme aided the system in adjusting the spatialization in accordance with the user's analytical reasoning. Finally, how did users interact with the system during their analytic process (i.e., were they focused on adjusting the weighting scheme, or focused on synthesizing information)?

Our results show that each user's weighting scheme was updated in accordance with that user's reasoning at specific times during the investigation, and that it provided the flexibility for this scheme to adapt to the dynamic process of each user. The updates to the spatialization based on semantic interaction provided support for each user's process, such as suggesting which documents to read next, incrementally determining the meaning of a cluster, and promoting the re-visiting of information. Users conducted their investigation by utilizing the semantic interactions to synthesize information, without focusing directly on the weighting scheme. The final spatializations generated in ForceSPIRE were co-created by the user and the system, as evidenced by a mixture of user-defined document locations, and model-defined locations, and were representative of the findings of the user as evidenced by their debriefing. These positive results suggest that semantic interaction in ForceSPIRE provides meaningful computational support for sensemaking. As a result, users are able to focus on the synthesis of information in the spatialization, while the system provides computational foraging support suited to the user's analytic process. 


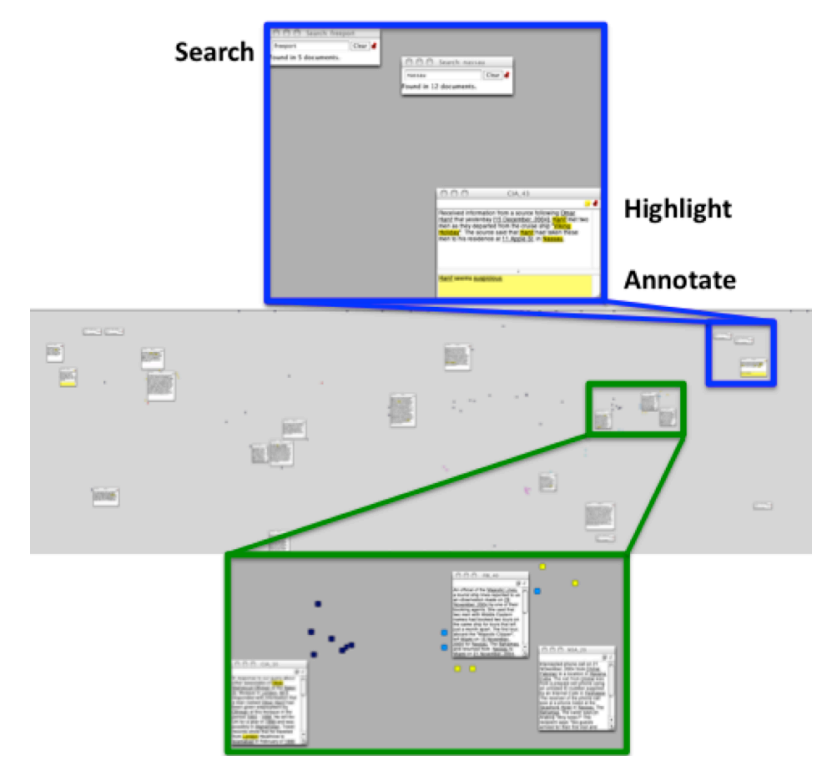

Fig. 1. A scaled-down screenshot of ForceSPIRE taken on the large, high-resolution display used in this study (two zoomed in views shown). Users can search, highlight, annotate, and reposition documents spatially. Documents can be shown as minimized rectangles, as well as full detail windows.

\section{Related Work}

Sensemaking is an inherently cognitive-demanding task. It involves establishing implicit connections between information based on the domain expertise and intuition of the user. Pirolli and Card [3] model this process as a sequence of cognitive stages pertaining to foraging (i.e., collecting and filtering information) and synthesis (i.e., generating and testing hypotheses and relationships) [6]. It is important to note that while this sensemaking loop contains specific stages, sensemaking as a process is very complex, and at times these stages are cognitively performed in parallel [7]. Therefore, we believe it is an important goal of visual analytic tools to support this dynamic process by coupling foraging and synthesis.

Spatializations have been used to support foraging by representing high-dimensional information, such as text, in an easily comprehendible two-dimensional view. In such views, the primary representation is one where information that is relatively closer to other information is more similar [5], enabling users to find relevant information and gain new insights. For example, the "Galaxy View" within IN-SPIRE [8, 9] presents users with a spatial clustering of documents based on a dimension-reduction algorithm. Users can interact and modify this view via direct manipulation of the keyword weighting (i.e., if a user finds a term more important, she can increase the weight on that term). Similarly, STREAMIT enables direct control over keyword weighting in a force-directed model [10]. Visualizations such as "Dust \& Magnet" [11] and VIBE [12] allow users to place specific points of interest in the space, acting as anchors from which the remaining points attract and repel form.

Spatializations have also been used to support synthesis by enabling users to externalize their insights during an investigation. In a spatial workspace where users can manually manipulate the location of information, users build spatial structures to capture their synthesis of the information over time - a process referred to as "incremental formalism" [13-15]. Andrews et al. found that intelligence analysts can make use of such spatial structures as a means to externalize insights during sensemaking, manually placing relevant documents in clusters on a large, high-resolution display
$[16,17]$. Additionally, they found that the large display workspace promoted a more spatially-oriented analysis. Tools, such as Analyst's Notebook [18], Jigsaw's "Tablet" [19], nSpace2 [20], Analyst's Workspace [21], and others have also found it helpful to provide users with a workspace where spatial representations of information can be manually organized. Semantic interaction strives to enable a similar ability, without requiring the user to manually place all the information. Instead, the goal is for the system to learn from the interactions and co-create the layout, essentially merging the foraging and synthesis uses of spatializations.

Developing user interactions for visualizations that integrate into the sensemaking process and support analysis is an open challenge for visual analytics [22]. For example, Green et al. mentioned that during an analysis, users become focused on their task, a state they call being in the "cognitive zone" [23]. They propose that it is the responsibility of designers to offer interactions that thus focus on the task (and the data), rather than on the tool, as a means for keeping users in their cognitive zone. Similarly, Elmqvist et al. presented the concept of "fluid interaction" as a way to maintain the "flow" of the analytic process [24]. They claim that interactions should be designed so as to adhere to the inherent flow of each individual's analysis. Dou et al. have demonstrated the capability for interaction to portray a user's reasoning process [25]. They showed that humans could interpret a log of another user's interactions during an analysis and effectively infer that user's reasoning. With semantic interaction, we aim to infer the user's reasoning systematically, supporting the "flow" of the investigation through updating the mathematical model while the user focuses on synthesizing.

\section{Semantic InTERAction In ForcespiRE}

ForceSPIRE (Fig. 1) is a visual analytic prototype for spatial text analysis using semantic interaction [1]. ForceSPIRE presents users with a spatialization of the dataset, representing documents as minimized boxes, which can be expanded to full-detail documents via double-clicking. The underlying force-directed model (modified from [26]) treats documents as nodes, and edges between nodes are created if one or more keyword entity co-occurs in both documents. Entities are algorithmically extracted using LingPipe [27]. The model learns through various semantic interactions that affect the weighting, creation, and removal of entities, and the "mass" of documents. An overview of the supported semantic interactions, their corresponding analytical reasoning, and coupling to the underlying force-directed model is shown in Table 1. ForceSPIRE also includes an "Entity Viewer", from which entities can be directly created, removed, as well as have their weights directly modified. ForceSPIRE supports the following semantic interactions (described in more detail in [1]):

Document movement allows users to manipulate the spatial layout directly in the spatialization by placing documents in locations based on the user's domain knowledge. These movements can be both exploratory and expressive [28]/\{v2pi/\}, differentiated by how they adjust the underlying model. Exploratory movements do not change the weighting of keywords (or entities), but use the current weights to determine the position of the remaining documents given the user-defined location of the document being moved. These can be seen as a "model constraint", as the user decides the placement of one or more documents, and the model produces the remaining layout based on these static locations. With expressive movements, users are able to inform the system that the weighting scheme should be updated to reflect the increased similarity between two (or more) documents. For example, when placing two documents closer together, the system determines the similarity between those two documents, and increases the weight on the corresponding entities. As a result, a new layout is incrementally generated reflecting the new similarity weighting, where those two documents (as well as others sharing similar entities) are closer together. Users also have 
Table 1. Forms of semantic interaction supported in ForceSPIRE. Each interaction corresponds to reasoning of users within the analytic process. Corresponding model updates are performed to steer the model based on the user's reasoning.

\begin{tabular}{|c|c|c|}
\hline Semantic Interaction & Associated Analytic Reasoning & Model Updates \\
\hline Document Movement & $\begin{array}{l}\text { - Similarity/Dissimilarity } \\
\text { - Create spatial construct (e.g. cluster, timeline, list, etc.) } \\
\text { - Test hypothesis, see how document "fits" in region }\end{array}$ & $\begin{array}{l}\text { - Similarity/Dissimilarity b/w documents } \\
\text { - Up-weight shared entities, down-weight } \\
\text { others }\end{array}$ \\
\hline Text Highlighting & $\begin{array}{l}\text { - Mark importance of phrase (collection of entities) } \\
\text { - Augment visual appearance of document for reference }\end{array}$ & - Up-weight highlighted entities \\
\hline $\begin{array}{r}\text { Annotation, "Sticky } \\
\text { Note" }\end{array}$ & $\begin{array}{l}\text { - Put semantic information in workspace, within document } \\
\text { context }\end{array}$ & $\begin{array}{l}\text { - Up-weight entities in note } \\
\text { - Append entities to document and model }\end{array}$ \\
\hline $\begin{array}{r}\text { Level of Visual Detail } \\
\text { (Document vs Icon) }\end{array}$ & $\begin{array}{l}\text { - Change ease of visually referencing information (e.g. full } \\
\text { detail = more important = easy to reference) }\end{array}$ & $\begin{array}{l}\text { - (Full Document): "heavier node", } \\
\text { increase node's friction } \\
\text { - (Icon): "lighter node", less friction }\end{array}$ \\
\hline Search Terms & - Expressive search for entity & $\begin{array}{l}\text { - Up-weight entities contained in search } \\
\text { - Add entities to model }\end{array}$ \\
\hline
\end{tabular}

the ability to pin documents to specific locations. These documents serve as spatial landmarks, in that they persist at that location, and the force-directed model treats them as layout constraints, organizing the remaining documents around them. Additionally, pinning allows ForceSPIRE to distinguish between exploratory and expressive movements. Dragging a document near a pinned document will briefly color both documents pink to alert the user of the expressive movement (if the user releases the document at this location). Thus, all other movements in the space are exploratory movements.

Text highlighting allows users to highlight text segments directly in the full-detail document views. As a result, the system increases the importance of the terms highlighted, updating the underlying mathematical model, and ultimately the layout. The phrases highlighted are also parsed for entities using a more aggressive entity extraction algorithm, so as to add entities to the model that may have been initially missed. (Users also have the ability to select an exact entity to add to the system.)

Search allows users to perform a standard text search within the dataset. As a result, documents containing the search term will be highlighted, and an edge between the search box and those documents will be created (multiple search boxes can exist). The model is updated by increasing the weight of the entity searched for (and creating a new entity for the search term if it does not already exist).

Annotation is available for each document in the dataset. Through annotating a document, users can add "meta-information" to the document based on their domain expertise. For example, adding a note "relates to the events in Chicago" results in parsing the note for entities (i.e., "Chicago"), and adding them to the document, which creates edges to other documents containing "Chicago".

Each of these semantic interactions creates soft data, a quantitative representation of captured user interaction within the context of the dataset. Fig. 2 models how soft data is collected (i.e.,

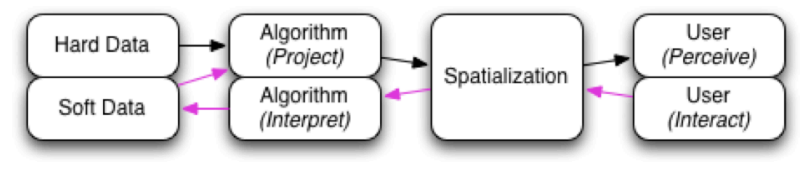

Fig. 2. The spatialization in ForceSPIRE is treated as a "medium for interaction", where interpreted analytical reasoning from each interaction is stored as "soft data" [1]. captured and interpreted interactions within the context of the dataset), as well as how it is combined with the hard data to produce the spatial layout. As a result, the soft data steers the underlying force-directed model. Also, soft data serves as a log of the entity weighting throughout the user's analytic process, and can be examined at any time to gain insight about their process.

\section{Method}

This user study investigates the following research questions about the capabilities and benefits of semantic interaction:

1. How well can semantic interaction systematically quantify analytical reasoning based on user interaction as a dynamic entity-weighting scheme?

2. How does the real-time modification of the weighting scheme and adjustment of the spatialization aid users' sensemaking?

3. What was the focus of users while exploring the dataset through semantic interaction? That is, were they focused on adjusting the weighting scheme, or synthesizing information?

4. How does the co-created spatialization map to the users' findings?

We hypothesize that the coupling between the semantic interactions and model updates will create a dynamic weighting scheme that appropriately captures a signature of the analytical reasoning of each user throughout his or her investigation in the form of entity weights. As a result, this weighting scheme will adjust the spatialization, aiding in the co-creation of the layout, where users need not develop the entire layout manually, but also not rely on solely algorithmic generation. During this process, this will help users by adjusting the layout while users read documents and synthesize the information, bringing related documents nearby. Also, we hypothesize that the soft data captured during the analysis will be representative of the analytic product of each user, and therefore the co-created spatialization will be meaningful to the user. Throughout this process, we hypothesize that the users will remain focused on the synthesizing of information, rather than interacting to directly modify the weights of entities.

\subsection{Equipment}

For this study, we used a large, high-resolution display (shown in Fig. 3). Such workstations allow users of ForceSPIRE to leverage the additional resolution to show many text documents at full detail, and the additional physical size to provide users with a more embodied analytic experience $[17,21,29,30][31,32]$. This particular 


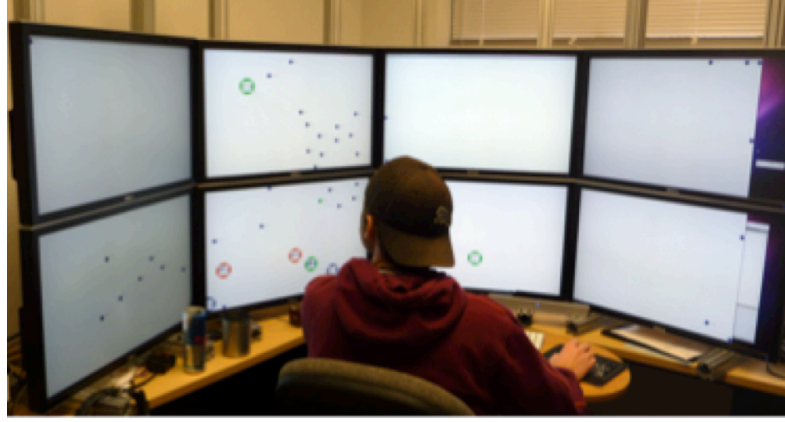

Fig. 3. The 32 megapixel large, high-resolution display used in this study.

workstation is constructed using 8 30-inch displays, driven by a single node, providing a total workspace resolution of $10,240 \mathrm{x}$ 3,200 pixels. The curvature allows easy access to all areas via physical navigation, such as chair rotation $[32,33]$.

The dataset used for this study is an analysis exercise called Atlantic Storm developed for the purpose of training and evaluating intelligence analysts, as well as analytic tools. The dataset consists of 111 text intelligence reports containing a fictitious terrorist plot. Using LingPipe [27] to extract keywords (i.e., entities) from these documents, 294 unique entities occurring more than once in the dataset were extracted (singletons were removed). The choice to use this dataset is based on the ability to have a realistic dataset, containing a known ground truth against which to compare the findings of the users, while requiring no detailed domain knowledge beyond English reading comprehension and creativity.

\subsection{Data Collection and Analysis}

ForceSPIRE has the ability to log the soft data used for semantic interaction. For the purpose of this study, this gives us a record of every interaction performed by the user, as well as how the system interpreted the interaction in context of the dataset. For example, when a user highlights a phrase, the soft data shows us when the highlight occurred, what the text is, in which document, as well as what entities' weights changed, and what the new weights are.

The users were asked to provide us with verbal feedback throughout their process. In a post-study interview, subjects explained their findings, the resulting spatialization, and insights about their process that may have been missed during the think-aloud protocol. Video recordings and screenshots were also taken during each task for post-study analysis.

\subsection{Procedure}

This study consisted of observing 6 computer science graduate students. The age of the participants ranged from 27 to 38, with an average age of 30 .

Each participant was given a brief overview of ForceSPIRE, using a practice dataset, for the purpose of making each user familiar with how ForceSPIRE and the supported semantic interactions function. Upon informing us that they were comfortable, each user was given verbal instructions on their task. Each user was given the same initial view of the Atlantic Storm dataset in ForceSPIRE as a starting point. We informed the participants that they had a maximum of 90 minutes to analyze the dataset, after which they will be debriefed regarding their investigation. They were allowed to finish early if they felt they were finished before time expired.

\section{Results}

The success of a visual analytic tool hinges on the ability to provide support during the analytic process, as well as a meaningful representation of the user's findings. Thus, the results of this study
Table 2. Semantic interaction counts during each user's analysis.

\begin{tabular}{|r|c|c|c|c|c|c|c|}
\hline \multicolumn{10}{|c|}{ User } \\
\hline Interaction: & $\mathbf{1}$ & $\mathbf{2}$ & $\mathbf{3}$ & $\mathbf{4}$ & $\mathbf{5}$ & $\mathbf{6}$ & Total \\
\hline Search & 13 & 32 & 37 & 14 & 38 & 21 & 155 \\
\hline Highlight & 47 & 58 & 12 & 10 & 5 & 0 & 132 \\
\hline $\begin{array}{r}\text { Expressive } \\
\text { Movement }\end{array}$ & 45 & 76 & 47 & 62 & 26 & 27 & 283 \\
\hline $\begin{array}{r}\text { Exploratory } \\
\text { Movement }\end{array}$ & 41 & 102 & 64 & 26 & 98 & 43 & 374 \\
\hline Annotation & 3 & 40 & 3 & 0 & 0 & 0 & 46 \\
\hline Total & 149 & 308 & 163 & 112 & 167 & 91 & \multicolumn{5}{|c}{} \\
\hline
\end{tabular}

are presented in terms of the analytic process and product. The analytic process describes how the semantic interactions within ForceSPIRE were used during the analysis, how the corresponding model and spatialization updates benefitted the users, and how the soft data mapped to each user's process. The analytic product details how the findings of each user are represented in the final spatialization, as well as the final weighting of keywords.

\subsection{Analysis of Process}

Each user's process was different, and thus utilized semantic interaction differently (Table 2). However, the analysis of each user's process reveals general usages of each semantic interaction. To address the research questions, we present the analysis of the processes of the users in terms of usage (how and when they used each semantic interaction), reasoning (what was their purpose for interacting, sensemaking or model steering), impact on weighting scheme (how the updated weighting scheme coincided with their reasoning process), and impact on spatialization (how did the updating spatialization benefit their analysis).

\subsubsection{Semantic Interaction Usage}

Performing a spatial analysis of data focuses around rearranging documents and creating spatial constructs or clusters [17, 34]. As such, pinning and document movement (both exploratory and expressive), were the fundamental methods of exploring the dataset. Pinning documents to absolute positions in the spatialization was used to create "spatial landmarks". That is, users pinned a document to a specific location in the layout to create (and maintain) meaning of a specific region of the spatialization. Based on these landmarks, document movement was used to organize the spatialization based on the user's intuition. For example, User3 pinned a document mentioning "Nassau" in a specific location. From there, he placed other documents related to "Nassau" nearby, and also quickly reacquired these documents when needed.

Highlighting was used mostly while reading a document to indicate terms or phrases that "stood out". These highlights were beneficial to users to produce visual and cognitive aids. The highlighted phrases (mostly single words and fragments of sentences) helped remind users of what information was important in a document when re-acquiring the document later. User6 was the only user who did not perform any highlighting during his investigation, simply stating that he "did not feel a need to."

Search was used to find other documents containing a term of

Table 3. Number of entities added via semantic interaction during each user's investigation. The majority of these new entities (92\%) maintained a weight above 0 throughout their process.

\begin{tabular}{|r|c|c|c|c|c|c|}
\cline { 2 - 7 } \multicolumn{1}{c|}{} & \multicolumn{6}{|c|}{ User } \\
\cline { 2 - 7 } & $\mathbf{1}$ & $\mathbf{2}$ & $\mathbf{3}$ & $\mathbf{4}$ & $\mathbf{5}$ & $\mathbf{6}$ \\
\hline Entites Added & 43 & 62 & 35 & 13 & 15 & 10 \\
\hline Weight > 0 & 38 & 54 & 35 & 13 & 14 & 10 \\
\hline
\end{tabular}




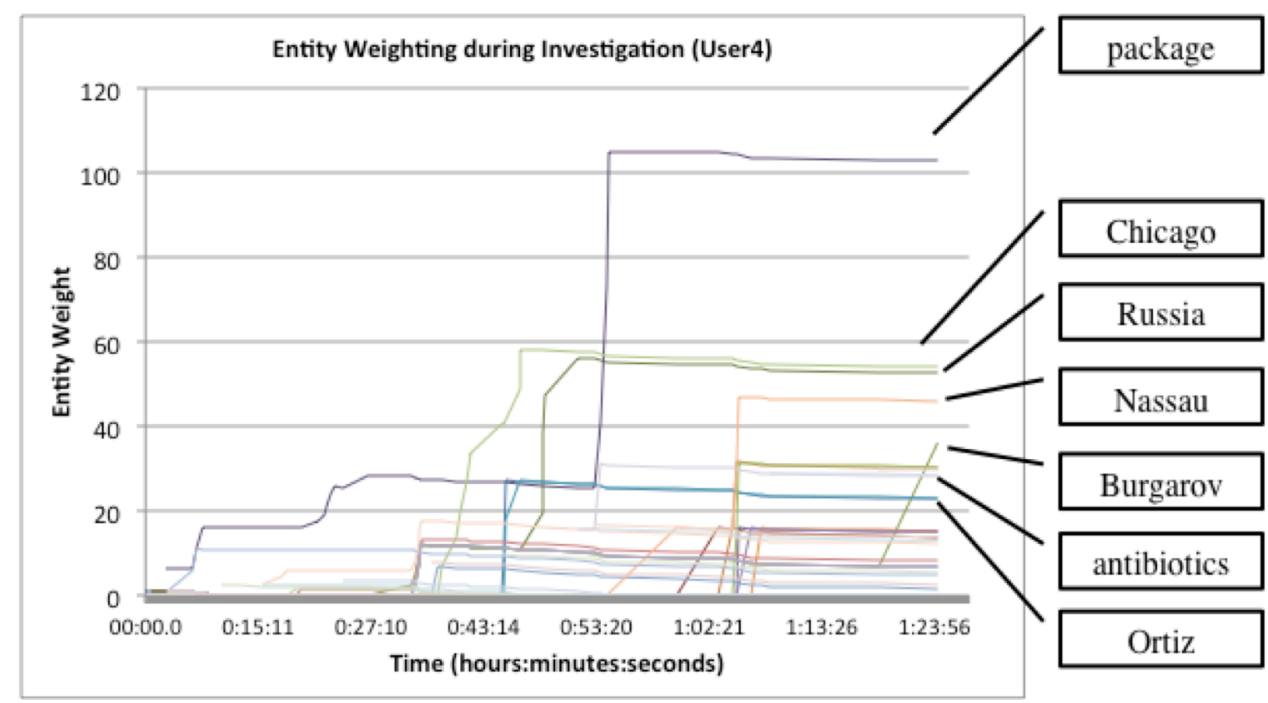

Fig. 4. User4's entity weighting over the duration of his analytic process. Semantic interactions in ForceSPIRE adjusted the weights of entities to coincide with his investigation of multiple hypotheses. As a result, the layout adjusted incrementally with each interaction.

interest to the user. Generally, users performed a search on keywords for two reasons. First, the unique color assigned to each search provided a quick overview of where in the dataset the term occurred given the current spatial layout. Second, users treated the search window (of which more than one could be opened) as a means to "tag" the space. For example, all of the users commented that leaving multiple search windows open and pinned to specific locations was an effective way to recall the meaning of that specific position in the spatialization.

With the exception of User2, annotation was rarely used. User2 said that he enjoyed the ability to "add personalized notes to important documents." In his case, ForceSPIRE detected 23 entities in his annotations (that were not in the dataset), including entities such as "irrelevant", "suspicious", and "revisit" (extracted from a note stating "should revisit this later"). During his investigation, he also found it useful to track what documents he found important by scanning the workspace and seeing which documents had the yellow notes window visible. Thus, annotations can be helpful to some users, while others prefer to utilize other interactions to support their analysis.

\subsubsection{Aiding the Sensemaking Process}

The primary benefit for sensemaking provided by ForceSPIRE was aiding the user in adjusting the layout by bringing related information nearby. Each semantic interaction in ForceSPIRE is tightly coupled with the dynamic weighting scheme used by the force-directed model responsible for generating the spatial layout. As such, ForceSPIRE responds to each interaction via updating the spatialization as a result of the updated weighting scheme.

Each user's process involved multiple stages of the investigation, including exploring specific leads (e.g., a person, place, etc.) and hypotheses regarding the dataset. As such, it is important for semantic interaction to allow a flexible entity weighting scheme to support exploring each of these aspects during different times of the investigation. For example, while a user investigates information regarding the entity "Atlanta", the weight of entities similar to (and including) "Atlanta" should increase. If the user chooses to investigate "weapons" at a later time, the weighting scheme should reflect this change. The challenge then, comes in supporting the rapid and fluid change of what is currently being investigated by a user through rapidly changing keyword weights, while maintaining a history of the previously emphasized keywords.

For example, Fig. 4 shows the temporal history of User4's keyword weighting during his analysis. The patterns and trends observed in User4's analysis of the soft data is also representative of the other users' history. One can see that approximately two minutes into the investigation, the entity "package" was created. The creation occurred while User4 read a document and found the phrase "carefully wrapped package" important, and thus highlighted it. The effect on the layout was that documents containing the entity "package" were brought closer. He did not immediately switch to reading those documents, instead continued to read the document while other related documents came nearby. This strategy was found in other users' processes also. "It was nice to see what documents would come near while I was reading and highlighting", User1 told us after his investigation. He continued to tell us that he would notice other documents coming closer, but would "continue reading and highlighting until I finished that document, then decide where to go next depending on what's close by." Upon finishing reading the document, User4 pinned it, and chose the closest document to continue his investigation. This document was one related to "package", and important to the plot. He continued reading three

Table 4. Each user's top 5 entities, collected both from the user's debriefing (user), and based on the final entity weighting (model). Underlined entities indicate a match between the user and model. Bold entities were entities added to the model as a result of semantic interaction during the analytic process (i.e., missed by the initial entity extraction).

\begin{tabular}{|c|c|c|c|c|c|c|c|c|c|c|}
\hline 1 (user) & $\mathbf{1}$ (model) & 2 (user) & 2 (model) & 3 (user) & 3 (model) & $\mathbf{4}$ (user) $\mathbf{4}$ (model) & 5 (user) & 5 (model) & 6 (user) & $6($ model $)$ \\
\hline diamonds & diamonds & package & $\underline{\text { Nassau }}$ & explosives & Nassau & diamonds package & Al Queda & Nassau & diamonds & weapons \\
\hline scholarship & weapons & $\underline{\text { Hanif }}$ & $\underline{\text { Hanif }}$ & weapon & students & Nassau & Caribbean & Miami & antib & nds \\
\hline $\mathrm{ji}$ & gra & antibiotics & Fre & $\underline{\mathrm{Na}}$ & weapons & Bur & $\mathrm{Ru}$ & $\mathrm{rt}$ & 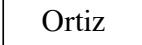 & $\mathrm{u}$ \\
\hline weapons & Jamal & diamonds & Miami & students & sch & ics Nassau & Hanif & $\mathrm{A}_{1}$ & Bahamas & ate \\
\hline freeport & Nassau & $\underline{\text { Nassau }}$ & package & scholarship & Jan & ge Bur & Odeh & we & $\underline{\text { Hijazi }}$ & $\underline{\mathrm{Hi}}$ \\
\hline
\end{tabular}


more documents containing "package", highlighting other phrases that contained the term. As such, the term continued to increase in weight, and related documents continued to form more tightly around the one that was pinned.

Fig. 4 also shows instances when User4 explored other potentially relevant information. For example, at 36 minutes into his analysis, he informed us that he wanted find out more information regarding events in "Chicago". This stemmed from reading a document that mentioned "Chicago". He highlighted the single word, and immediately pinned the document in a specific location, away from other documents. Then, he searched for the term "Chicago", and placed the search window next to the pinned document. Then, he opened and read some of the documents containing Chicago (they were highlighted teal from the search) that came closer. The first 2 documents he read, he placed near the first pinned document. As a result, the weighting of "Chicago" increased, but so did the weights of other related entities, such as "Russia", "weapons", and "Panama City". This occurred because the documents he dragged near the pinned document containing "Chicago" were not similar based on only "Chicago", but those other entities as well. As a result, more documents came closer that did not contain strictly "Chicago", but were related. In this case, he read some of the documents containing "Russia" and "weapons" and moved them into another, separate location. Again, ForceSPIRE responded by moving the documents more similar to "Russia" and "weapons" into that location, rather than near the location regarding "Chicago". This benefitted the user as he noticed how some documents remained in the middle of the two areas, showing relevance to both topics. These were documents connecting these two, and important to the plot.

Towards the end of his investigation (approximately one hour into it), he focused on tying all the pieces of evidence he had collected together. He did so through exploring the relevance of "Bahamas" and "Nassau". He did so primarily through small, local movements within an area specific to each. He arranged the documents within the region to reflect a sequence of events related to transportation of a package. In addition to the weight increase of those entities, he discovered the relevance of some of the key persons involved in the suspicious activity (i.e., "Odeh", "Hanif", and others). He found an important document detailing how some of the weapons (which he investigated earlier) were possibly being transported by students funded through a suspicious scholarship fund. The history of his weighting scheme reflects each of these hypotheses and branches during his investigation, as indicated in Fig. 4.

In addition to increasing the weighting of entities that were relevant to the investigation, semantic interaction also reduced the weight of entities that were not. ForceSPIRE does so through "decaying" the weights of previously emphasized keywords over time. That is, as other keywords are emphasized via various semantic interactions, weights of previously emphasized keywords will begin to decay. Therefore, if they are never investigated again, they will eventually return to their lower weight, but if they are revisited again later, they will increase in weight again. At times, this resulted in the weights of those entities going to zero (thus having no impact on the spatial layout). Across all users' processes, the average number of entities where this occurred at least once is 245 (out of the 294 unique entities initially extracted by ForceSPIRE). While these entities did not have an impact on the spatial layout when their weight was set to 0 , subsequent semantic interactions continued to use these entities to measure similarity. As a result, entities that may not have been relevant during the early stages of an investigation and were relevant to a later hypothesis being explored, saw their weight increased. An example from User4 is the term "Nassau", which was not relevant to his investigation until approximately 54 minutes into the study, where the weight increased from zero (as shown in Fig. 4). This happened as a result of him dragging one document close to another on the basis of both being about an event in the "Bahamas".

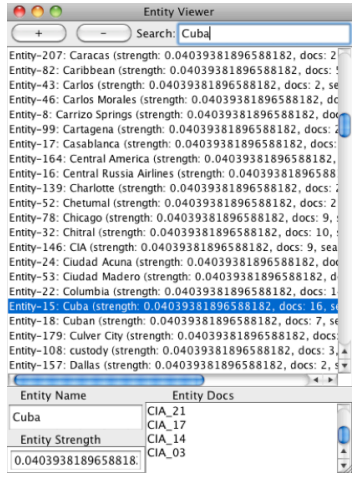

Fig. 5. The "Entity Viewer" in ForceSPIRE allows users direct control over entity weights, adding entities, and removing them. With semantic interaction, this view was not needed.

ForceSPIRE interpreted this similarity, but also found these documents similar because of "Nassau", increasing the weight of the term and bringing those related documents nearby.

Semantic interaction aided two users from this study in creating a "junk pile" (i.e., a collection of documents that are not relevant to the main plot, and are thus placed in a location away from the relevant information). As these two users placed more information into the same cluster that they referred to as "junk", ForceSPIRE calculated the similarity between the documents being placed into this cluster and increased the weight of those entities. "Look! It's moving other junk into my junk pile for me" User1 remarked. However, he was sceptical of the system's ability to detect irrelevant documents, so he opened and read a few of them as they moved closer. Some, he agreed with being junk and left them in the junk cluster, while others he moved near other pinned documents in the spatialization. By doing so, he continued to improve ForceSPIRE's ability to detect irrelevant documents. When asked about this experience after the study, he told us that the more he interacted with the layout (including his "junk pile"), the more pleased he became with the metrics for determining junk, and the "more [he] trusted it".

An important capability in ForceSPIRE is the steering of the entity extraction algorithm for generating additional entities during an investigation. Entities can be added to the system through semantic interaction, which was critical to the ability to capture and infer the users' reasoning processes. While the entity extraction algorithm in ForceSPIRE managed to extract 294 unique entities, each user found additional entities that were relevant to their analysis. Table 3 shows the number of entities created as a direct outcome of semantic interaction. Of these, most (92\%) maintained a weight greater than zero throughout the investigation. This shows that not only was it important for users to steer the weighting of existing, extracted entities, but also to steer the entity extraction algorithms to generate additional entities. For example, User3 highlighted the phrase "he has students now in the USA", which was passed through a more aggressive entity extraction algorithm, and detected "students" as an entity. This entity was important to the user's findings, as well as highly weighted in the model (Table 4).

Pinning and un-pinning documents was used not only to place meaningful documents in absolute positions in the workspace, but also to check if the current weighting model would place the document in another region (or into another cluster). For example, three of the users commented that they un-pinned a document to see where it went after it had been pinned for a long time. They were interested in other possible topics it might relate to. If nothing particularly interesting was found, they returned the document to the previous location and pinned it again. However, often users found relationships between these documents and other clusters, and typically either left the document un-pinned, or pinned it in a different location from where it was pinned previously. For instance, 
Table 5. Pinned and unpinned documents and search windows in each user's final spatialization.

\begin{tabular}{|c|r|c|c|c|c|c|c|}
\cline { 3 - 8 } \multicolumn{2}{c|}{} & \multicolumn{7}{c|}{ User } \\
\hline \multirow{4}{*}{ Docs. } & Pinned & 34 & 41 & 44 & 28 & 32 & 34 \\
\cline { 2 - 8 } & Un-Pinned & 77 & 70 & 67 & 83 & 79 & 77 \\
\cline { 2 - 8 } & Detail & 85 & 88 & 89 & 36 & 40 & 49 \\
\cline { 2 - 8 } & Minimized & 26 & 23 & 22 & 75 & 71 & 62 \\
\hline Search & Pinned & 9 & 19 & 31 & 2 & 16 & 5 \\
\cline { 2 - 8 } Windows & Un-Pinned & 0 & 0 & 0 & 1 & 1 & 0 \\
\hline
\end{tabular}

User1 found that he had a document pinned from very early in his investigation that referred to the Freeport Star Hotel. When he unpinned it, he saw it go near other documents about the Bahamas and Nassau, which helped him make the connection about the events happening in that area.

In general, users emphasized the importance of observing the spatialization adjust incrementally. That is, to notice the change in relative distances between documents as a result of the highlighting they did while reading, searching, etc. Such exploration can be found in other tools, such as VIBE [12] or Dust\&Magnet [11], where users can place "points of interest" corresponding to keywords in specific locations, and observe how the spatial layout adjusts given those keywords and locations.

Users did not treat the semantic interactions as a means to directly manipulate entities. That is, they interacted as a means to synthesize the information. For instance, based on their comments, highlighting was performed not to pass a phrase through a more aggressive entity extraction algorithms, but to emphasize a part of the text as being important, so as to be able to find it again more easily later. "Oh, that's important ... [I] might need to come back to [it]", one user stated while highlighting a phrase. None of the users found the need to directly manipulate entities (e.g., adjust the weights, add, remove) via the "Entity Viewer" (shown in Fig. 5). All users were shown this feature in the training, but none found it necessary to use during their actual investigations. These results evidence that semantic interaction properly coupled the semantic interactions with model updates, to the extent that users never felt the need to do so directly. This contrasts with the intended usage of other tools, such as INSPIRE [35], where model steering occurs via direct parameter manipulation on the part of the user. With ForceSPIRE, users were successfully able to focus on the synthesis necessary for sensemaking, while the parameter adjustments occurred systematically in accordance with their analytic reasoning.

\subsection{Analysis of Product}

At the conclusion of each trial, we asked the user to describe his findings (both in terms of what information is relevant to the suspicious plot, which all users found, and information that was not). ForceSPIRE remained visible during this debriefing, but we asked users to not interact with the tool, but simply use the final layout as a means to help describe their findings. The analytic product with regards to this study refers to the final spatial layout in ForceSPIRE, as well as the user's debriefing after the study. We analyze this information in addition to the final weighting scheme (i.e., soft data) at the conclusion of the analysis. Compared to the known ground truth of the dataset, each user found the suspicious activities, with varying amounts of detail to support the findings. Thus, the analysis of the product here is with regards to ForceSPIRE's ability to create synergy between what the system and the user knows (rather than compared to the ground truth).

The final weighting schemes at the conclusion of each user's investigation served as a good approximation of the keywords
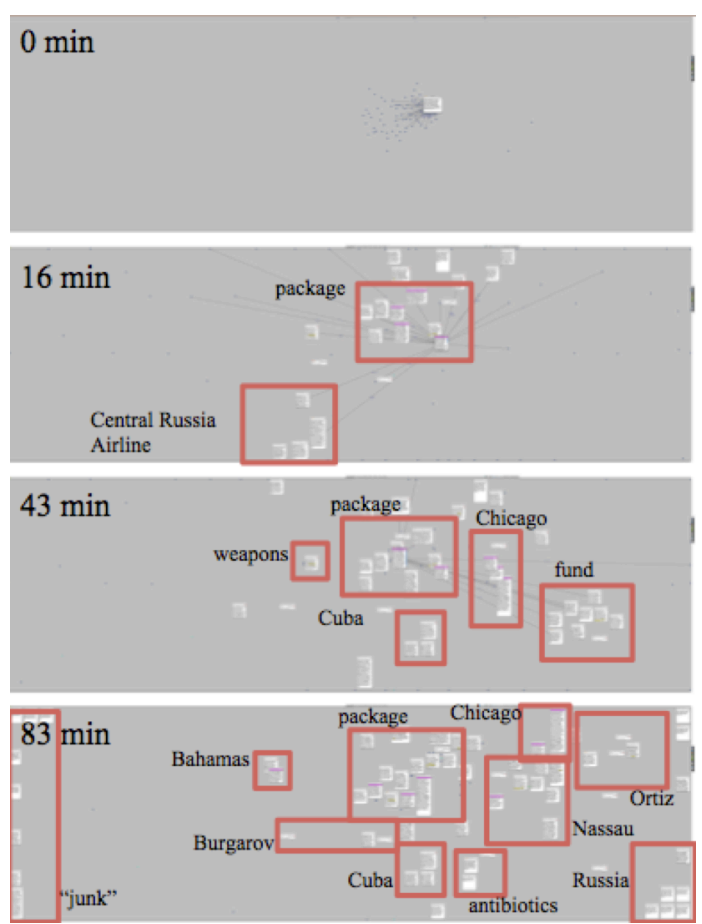

Fig. 6. The progression of the spatialization over time for User04. The annotations (labels and red region boundaries) were added after the study. They represent the meaning of the regions of the space, indicated by the user during his process.

relevant to their findings (see Table 4). This table shows the top 5 keywords from each user's debriefing and the top 5 entities based on entity weight, labelled "user" and "model" respectively. The entities from the debriefing were given to us by the user as part of their debriefing to represent their findings. The entity weights were obtained by taking the top 5 highest weighted entities in the final soft data state. The entities highlighted in bold are entities that were not initially extracted, but added to the model through the user's semantic interaction during the study.

These results reveal that $47 \%$ of the entities match directly from the user's findings to the highly weighted entities. In addition, 11 of the highly weighted entities were added to the model as a result of semantic interaction. Of these 11,7 were important based on the debriefing of the user (i.e., they matched with the top 5 entities given to us by the user). Therefore, not only were these entities added as a result of semantic interaction, but they were also relevant to the user's findings.

In some cases, there is not a direct match between the entities obtained during the debriefing and the entity weighting. For example, User5's entities show no direct matches. However, a more sophisticated entity correlation algorithm may find connections between entities such as "Caribbean" and "Nassau", "Freeport", "Miami", and "Apple St." (an address in Nassau in this dataset). Thus, even when there are not direct matches, we find that the higher weighted entities provide a good estimate of characteristics of the dataset users found important and relevant to their investigation. In fact, this indicates that the system was successfully able to interpret the user's reasoning within the context of the actual data. That is, the system identified keywords relevant to the user's process that the user did not think of or at least had used other words in place of. This suggests that the system fulfills the needs of incremental formalism and ill-defined user-generated clustering [34].

\subsubsection{Spatialization Co-Creation}

One of the goals of semantic interaction is to properly steer the underlying model to allow for co-creation of the spatialization 
between the user and the model. As such, we hypothesized that some documents (and search windows) would be pinned by the user to maintain their absolute position in the workspace, and others would be un-pinned so that the model could determine their position based on the current entity weighting. Table 5 shows the number of pinned and un-pinned documents and search windows in the final layout produced by each user.

In the final layouts, $32 \%$ of the documents were pinned. Based on the debriefing, the users informed us that some documents were pinned to maintain their absolute position in the workspace for the purpose placing meaning into the workspace. However, others were at times pinned for more detailed adjustments to cluster layout. For example, User2 commented that he would pin documents in a cluster so that he could create detailed spatial structures within a cluster (e.g., a timeline). ForceSPIRE currently does not well support such multi-scale spatial layouts, but feedback from users suggests doing so in the future.

The majority of search windows were pinned (98\%). Users treated them as "tags" for their spatialization, as searches were performed on entities. The only two users who had one search window un-pinned (User4 and User5), explained that they preferred to have it "float" to get an idea of where the documents are that related to that term.

For example, User4's progression of the co-created spatialization can be seen in Fig. 6. After 16 minutes, his layout was made up of two main clusters: one about "package" and one about the "Central Russian Airline". Then, as his investigation continued, he learned more about the dataset (e.g., a suspicious "fund", documents about "weapons", etc.). At the completion of his trial (83 minutes), he was aware of much more detail regarding the dataset, such as events happening in "Nassau" regarding the "package", a suspicious person named "Ortiz", and a unrelated plot in "Russia". Additionally, the layout placed a collection of documents along the far left, which the user told us were "junk". These results indicate that the spatialization was successfully co-created (based on the coinciding weights, shown in Fig. 4), and maintained meaning for the user. As such, not only did the weights reflect the analytic reasoning of the user, but the spatialization seemed to successfully reflect the shared knowledge between the user and the system at each stage.

\section{Discussion}

Through this user study, we explored and validated the principles grounding semantic interaction. These principles include (fully described in [1]:

- $\quad$ capturing semantics from user interaction;

- $\quad$ shielding the users from direct parameter manipulation;

- incrementally co-creating of the spatialization through incremental model learning, to coincide with the incremental formalism [13] of the user.

\subsection{Capturing Semantics from User Interaction}

ForceSPIRE captured the semantics from users in terms of the entities contained in the dataset. The method for capturing these semantics is supported via changing the weighting of entities, as well as the creation of entities. In turn, the semantics are reflected in the steering of the weighting scheme of the model.

The ability to steer this model at multiple levels of detail was important because it coincided with the user's reasoning about different levels of detail. For example, document movement allowed users to provide more broad and informal feedback regarding important relationships between documents. Generally, this feedback was at the document level, where users would create clusters of documents without a formalized schema as to precisely why they are all similar. For example, User 2 told us he enjoyed the flexibility of moving documents because "[the system] will eventually figure out what [he] mean[s]". Similarly, User4 used expressive movements more heavily than exploratory movements. When asked about his preference, he informed us that "[expressive movements] tell the system something", and that the more he told the system, the "better chance the system will figure it out".

Other semantic interactions, such as search and highlighting, were used to provide more detailed insight into what characteristics of a dataset were relevant to the user. Similar to the results found by Endert et al. [34], highlights were typically short phrases containing relevant entities, while searches were performed only on keywords. To the users, the highlighting was preformed to provide visual importance to portions of documents. However, the system interpreted these as indicators as to what information should be examined more closely, resulting in entity creation and upweighting. As a result, ForceSPIRE did not only change the distribution of the weights, given the initially extracted entities, to attempt to produce the best fit given the user feedback, but also added entities when given feedback regarding relevant text within the dataset. As shown in Table 4, the entities added through semantic interaction were not only highly weighted in the soft data, but many also correlate to entities important to the users.

\subsection{Shielding from Direct Model Steering}

The users in this study treated their investigation not as steering a model, but rather synthesizing information. This is an important distinction, as it shows semantic interaction as providing a fundamentally different and richer method of interacting with visual analytic systems. As a result, semantic interaction enabled a analytic process similar to the effective spatial processes described in $[13,16$, $34]$, where the informality of the spatial synthesis interactions were beneficial in supporting incremental formalism. However, unlike these examples where a majority of the spatialization is created manually, semantic interaction provides computational support. Therefore, while users realized an implicit ability for ForceSPIRE to learn about the characteristics important to them, the explicit use of the system was to synthesize information.

\subsection{Incremental Model Learning and Formalism}

Users develop insights into a dataset through interacting and exploring it. As such, users learn additional information as they proceed through their analysis. For example, when constructing spatial groups manually, the meaning of these clusters gradually changes as more information is learned [34]. A cluster that was created based on a single term of interest, may evolve to represent a more broad meaning, represented via a collection of terms.

ForceSPIRE was able to incrementally learn these insights, and translate them into representative entity weightings and ultimately helpful spatializations. For example, User4's cluster regarding "Nassau" transformed into a cluster also containing documents that did not include the term directly, but instead contained terms such as "Bahamas" and "Freeport Star Hotel". These were conceptually related events, and thus the user decided to group them. The structural relationship between these documents can be described as a "transitive relationship" [34] (i.e., there is a connecting document that contains both terms). One challenge in this form of relationship is determining which terms to use for the transitivity. ForceSPIRE incrementally learned these terms, through semantic interactions such as moving a document near a cluster (confirming membership) and moving a document towards a different cluster (disagreeing with transitive relationship).

\section{FutURE WORK}

The concept of "undoing" a semantic interaction is not trivial, as not only was the spatial location of a document adjusted, but the coupled model updates changed the entity weighting scheme used to probabilistically determine the position of the other documents. The version of ForceSPIRE used in this study did not include an undo 
functionality. However, none of the users requested it. User3 made an erroneous move, where he accidentally dragged a document near the wrong cluster. "Ooops, oh well, [ForceSPIRE] will fix that later", he said (instead of asking how to undo), implying that the small number of erroneous interactions will be outweighed by the sum of the meaningful interactions over time. One approach in another version of ForceSPIRE is to store previous weighting schemes, so that when an undo occurs, the previous weights are restored, and the model updates the spatialization accordingly [1]. However, a "true undo" of a semantic interaction would be one from which the model learns about the user's analytical reasoning. As such, instead of restoring the weights of upweighted entities from the interaction, the system could lower the weights below the previous weights to reflect the user's decision to discontinue the investigation of those topics. This is an open research area we plan to investigate.

The soft data captured during each user's process is used directly to steer the force-directed model calculating the spatialization (Fig. 2). We believe soft data has potential for additional benefits. Various types of biases are common pitfalls for analyses [36]. For example, confirmation bias can result in users accepting evidence to confirm a given hypothesis, and ignoring evidence that may refute it. Showing the history of weighting to users during their investigation might help illuminate some of these biases, making users more aware of the potential to explore other hypotheses. We are planning to incorporate a soft data graph view into the workspace in the future.

Soft data has the potential to aid a group of analysts with asynchronous collaboration. Each collaborator's soft data provides an approximation of both the process and the findings. For example, continuing an investigation started by another can be made easier by illuminating the process through observing the weighting changes. This gives the collaborator a better understanding of the hypotheses already explored. Additionally, when multiple analysts are asked to investigate the same dataset, the history of entity weighting for each user enables an overview of the group's collective investigation. One open challenge is how to effectively merge soft data from multiple users.

\section{Conclusion}

In this paper, we present results of a user study investigating the principles of semantic interaction for supporting sensemaking. Semantic interaction is an approach to user interaction with visualization that couples analytic interactions within a spatialization (e.g., document repositioning, text highlighting, search, annotations) with updates to the underling model responsible for generating the spatial layout. As such, semantic interaction tactfully combines interactions enabling users to synthesize information with model updates to support computational foraging support for sensemaking.

The results indicate that the semantic interactions in ForceSPIRE provided the flexibility for users to investigate and explore data spatially. Semantic interaction provided the expressiveness required to update the model to coincide with the user's analytic reasoning. The captured soft data during the investigation supports the analytic product of each user, and was also able to adapt to the different points of emphasis and hypotheses during each investigation. Users regarded semantic interactions not as direct model steering, but as interactions for synthesis. Finally, ForceSPIRE updated the spatialization based on the semantic interactions of the user, and the final layouts were representative of each user's findings. The spatialization was incrementally co-created between the user and the system.

Given the positive results of this study we encourage further research in this area to advance the field of visual analytics through exploring the science of interaction.

\section{ACKNOWLEDGMENTS}

This research was supported by NSF FODAVA grant CCF-0937071 and the DHS VACCINE Center of Excellence.

\section{REFERENCES}

[1] Endert, A., Fiaux, P. and North, C. Semantic Interaction for Visual Text Analytics. In ACM CHI, 2012.

[2] Thomas, J. J. and Cook, K. A. Illuminating the path. IEEE Computer Society, 2005.

[3] Pirolli, P. and Card, S. Sensemaking Processes of Intelligence Analysts and Possible Leverage Points as Identified Though Cognitive Task Analysis Proceedings of the 2005 International Conference on Intelligence Analysis, McLean, Virginia2005), 6.

[4] Endert, A., Fiaux, P. and North, C. Unifying the Sensemaking Loop with Semantic Interaction. In IEEE Workshop on Interactive Visual Text Analytics for Decision Making at VisWeek 2011, Providence, RI, 2011.

[5] Skupin, A. A Cartographic Approach to Visualizing Conference Abstracts. IEEE Computer Graphics and Applications, 222002), 5058.

[6] Pirolli, P. and Card, S. Information foraging in information access environments. In Proceedings of the SIGCHI conference on Human factors in computing systems, Denver, Colorado, United States, 1995.

[7] Kang, Y.-a. and Stasko, J. Characterizing the intelligence analysis process: Informing visual analytics design through a longitudinal field study. City, 2011.

[8] Wise, J. A., Thomas, J. J., Pennock, K., Lantrip, D., Pottier, M., Schur, A. and Crow, V. Visualizing the non-visual: spatial analysis and interaction with information for text documents. In Readings in information visualization: using vision to think, 1999.

[9] Bohn, S. J., Calapristi, A. J., Brown, S. D. and Nakamura, G. C. Analytic Steering: Inserting Context into the Informaion Dialog. In IEEE Workshop on Interactive Visual Text Analytics for Decision Making at VisWeek 2011, Providence, RI, 2011.

[10] Alsakran, J., Chen, Y., Zhao, Y., Yang, J. and Luo, D. STREAMIT: Dynamic visualization and interactive exploration of text streams. In IEEE Pacific Visualization Symposium, 2011.

[11] Yi, J. S., Melton, R., Stasko, J. and Jacko, J. A. Dust \& magnet: multivariate information visualization using a magnet metaphor. Information Visualization, 4, 4 2005), 239-256.

[12] Olsen, K. A., Korfhage, R. R., Sochats, K. M., Spring, M. B. and Williams, J. G. Visualization of a document collection: the vibe system. Inf. Process. Manage., 29, 1 1993), 69-81.

[13] Shipman, F. and Marshall, C. Formality Considered Harmful: Experiences, Emerging Themes, and Directions on the Use of Formal Representations in Interactive Systems. Comput. Supported Coop. Work, 8, 4 1999), 333-352.

[14] Shipman, F., Hsieh, H., Airhart, R., Maloor, P., Moore, J. M. and Shah, D. Emergent Structure in Analytic Workspaces: Design and Use of the Visual Knowledge Builder. City, 2001.

[15] Marshall, C. C., Frank M. Shipman, I. and Coombs, J. H. VIKI: spatial hypertext supporting emergent structure. In Proceedings of the 1994 ACM European conference on Hypermedia technology, Edinburgh, Scotland, 1994.

[16] Andrews, C. Space to Think: Sensemaking and Large, HighResolution Displays. Virginia Tech, Blacksburg, 2011. 
[17] Andrews, C., Endert, A. and North, C. Space to Think: Large, High-Resolution Displays for Sensemaking. In CHI, 2010.

[18] i2 Analyst's Notebook.

[19] Stasko, J., Goerg, C. and Liu, Z. Jigsaw: supporting investigative analysis through interactive visualization. Information Visualization, 7, 2 2008), 118-132.

[20] Wright, W., Schroh, D., Proulx, P., Skaburskis, A. and Cort, B. The Sandbox for analysis: concepts and methods. In $C H I$ 'O6, New York, NY, 2006.

[21] Andrews, C. and North, C. Analyst's Workspace: An Embodied Sensemaking Environment For Large, High-Resolution Displays. In IEEE VAST, Seattle, WA, 2012.

[22] Pike, W. A., Stasko, J., Chang, R. and O'Connell, T. A. The science of interaction. Inf Visualization, 8, 4, 263-274.

[23] Green, T. M., Ribarsky, W. and Fisher, B. Building and applying a human cognition model for visual analytics. Information Visualization, 8, 1 2009), 1-13.

[24] Elmqvist, N., Moere, A. V., Jetter, H.-C., Cernea, D., Reiterer, H. and Jankun-Kelly, T. Fluid interaction for information visualization. Information Visualization, 10, 4 (October 1, 2011 2011), 327-340.

[25] Dou, W., Jeong, D. H., Stukes, F., Ribarsky, W., Lipford, H. R. and Chang, R. Recovering Reasoning Processes from User Interactions. IEEE Computer Graphics and Applications, 292009), 52-61.

[26] Fruchterman, T. M. J. and Reingold, E. M. Graph drawing by force-directed placement. Software: Practice and Experience, 21, 11 1991), 1129-1164.

[27] Alias-i. 2008. LingPipe 4.0.1. City, 2008.

[28] Endert, A., Han, C., Maiti, D., House, L., Leman, S. C. and North, C. Observation-level Interaction with Statistical Models for Visual Analytics. In IEEE VAST, 2011.

[29] Andrews, C., Endert, A., Yost, B. and North, C. Information visualization on large, high-resolution displays: Issues, challenges, and opportunities. Information Visualization, 10, 4 (October 1 2011), 341-355.

[30] Endert, A., Andrews, C., Bradel, L., Zeitz, J. and North, C. Designing Large High-Resolution Display Workspaces. City, 2012.

[31] Ball, R., North, C. and A. Bowman, D. Move to improve: promoting physical navigation to increase user performance with large displays. In CHI 2007, San Jose, California, USA, 2007.

[32] Shupp, L., Andrews, C., Dickey-Kurdziolek, M., Yost, B. and North, C. Shaping the Display of the Future: The Effects of Display Size and Curvature on User Performance and Insights. HumanComputer Interaction, 24, 1 2009), 230 - 272.

[33] Endert, A., Fiaux, P., Chung, H., Stewart, M., Andrews, C. and North, C. ChairMouse: leveraging natural chair rotation for cursor navigation on large, high-resolution displays. In Proceedings of the 2011 annual conference extended abstracts on Human factors in computing systems, Vancouver, BC, Canada, 2011.

[34] Endert, A., Fox, S., Maiti, D., Leman, S. C. and North, C. The Semantics of Clustering: Analysis of User-Generated Spatializations of Text Documents. In AVI, 2012.

[35] Pak Chung, W., Hetzler, B., Posse, C., Whiting, M., Havre, S., Cramer, N., Anuj, S., Singhal, M., Turner, A. and Thomas, J. INSPIRE InfoVis 2004 Contest Entry, 2004.

[36] Heuer, R. Psychology of Intelligence Analysis, 1999. 